\title{
Impacto de las políticas de formación de maestros y evaluativas en el campo de la enseñanza de la filosofía en Colombia: 2014-2019*
}

Fecha de entrega: 27 de noviembre de 2019

Fecha de evaluación: 29 de enero de 2020

Fecha de aprobación: 10 de marzo de 2020

\author{
Maximiliano Prada Dussán** \\ Karol Nossa Caviedes ${ }^{* *}$
}

\section{Resumen}

Motivado tanto por el propósito nacional de mejorar los resultados en las evaluaciones estandarizadas internacionales, como por la firma del acuerdo de paz entre el gobierno nacional y las Fuerzas Armadas Revolucionarias de Colombia (FARC), en los últimos cuatro años el Ministerio de Educación Nacional de Colombia ha impulsado reformas relevantes que impactan directamente el campo de la enseñanza de la filosofía. Este texto describe las dos disposiciones normativas que más han llamado la atención de las comunidades filosóficas, en particular, y educativas, en general, en los últimos años: por un lado, las reformas a las licenciaturas, programas profesionales de formación de docentes, y, por otro, la reforma a la prueba de Estado Saber 11, que deben

* Este artículo es producto de la investigación "Estado del arte y balance del conocimiento construido en la Licenciatura en Filosofía de la Universidad Pedagógica Nacional en el período 2009-2018" (código DCs 507-19), adscrito y financiado por el Centro de Investigaciones de la Universidad Pedagógica Nacional. Citar como: Prada Dussán, M. y Nossa Caviedes, K. (2020). Impacto de las politicas de formación de maestros y evaluativas en el campo de la enseñanza de la filosofía en Colombia: 2014-2019. Cuadernos de Filosofía Latinoamericana, 41(123), 31-49. Dol: https://doi.org/10.15332/25005375/5988

* Profesor de la Licenciatura en Filosofía de la Universidad Pedagógica Nacional. Correo electrónico: aprada@pedagogica.edu.co

*** Estudiante de último semestre de la Licenciatura en Filosofia de la Universidad Pedagógica Nacional. Correo electrónico: IfI_kvnossac138@pedagogica.edu.co 
desarrollar los estudiantes de último grado de educación secundaria. A partir de este contexto, se sintetizan las reacciones que tales normas suscitaron en las comunidades académicas y, finalmente, se expresan los retos a los que la enseñanza de la filosofía se enfrenta a partir de tales cambios. Entre estos retos se encuentran la desprofesionalización filosófica del ejercicio docente y la reducción del saber filosófico a ejercicio de lectura.

Palabras clave: enseñanza de la filosofía, formación docente, pruebas Saber 11 .

\section{The Impact of Testing and Teacher Training Policies in the Field of Philosophy Teaching in Colombia: 2014-2019}

\section{Abstract}

In the course of the last four years, the Colombian Ministry of National Education - motivated both by the national intent to improve students' results in international standardized tests and by the signing of the peace agreement between the National Government and the Revolutionary Armed Forces of Colombia (FARC) - has promoted relevant reforms that directly impact the field of philosophy teaching. This text describes the two normative provisions that have attracted the most attention from philosophical communities in particular and of educational communities in general: the reforms of the bachelor in education degrees (professional teacher training programs) and of the Saber 11 official test, which all high school seniors must take before graduating. On the basis of this context, this article summaries the reactions that such norms aroused in academic communities and elaborates on the challenges that they pose to the teaching of philosophy. Some of these challenges are the philosophical de-professionalization of teaching and the degradation of philosophical knowledge to a reading exercise.

Keywords: Philosophy Teaching, Teacher Training, Saber 11 Test. 
La enseñanza de la filosofía ha cobrado interés recientemente entre distintos grupos académicos colombianos. Algunos de modo reactivo, ante la alarma que tanto las tendencias globales como las reformas gubernamentales locales han levantado sobre la presencia efectiva de la filosofía en la educación. Pero, también de modo activo, en la exploración de nuevos campos, estrategias, enfoques o didácticas de la filosofía. El proceso de paz, a su turno, ha lanzado un llamado que reclama a los filósofos su participación activa en este y otros acontecimientos relevantes en la historia nacional. Con todo, hemos sido testigos recientemente de un nuevo impulso entre la comunidad filosófica que busca involucrarse explícitamente con los eventos de interés público, especialmente, a través de estrategias que implican el trabajo colectivo e interinstitucional, más que individual o aislado.

En el campo de la enseñanza de la filosofía, hemos visto emerger en los últimos años un interés por el trabajo colectivo, por la renovación de las prácticas habituales de enseñanza y por la participación en escenarios de toma de decisiones de políticas públicas. Las recientemente conformadas Red Nacional de Programas de Filosofía, Red de Lógica y Argumentación, Red de Mujeres Filósofas, Red para la Formación Ética y Ciudadana, Red de Profesores de Filosofía, Comisión de Enseñanza de la Filosofía de la Sociedad Colombiana de Filosofía y el impulso que ha tomado la filosofía para niños, entre otras iniciativas, dan cuenta de este interés.

Dentro de este impulso, que presenta muchas aristas y perspectivas, en este artículo nos centraremos en mostrar el camino que ha recorrido la enseñanza de la filosofía en relación con las políticas públicas expedidas a nivel nacional recientemente, especialmente, a partir del Plan Nacional de Desarrollo 2014-2018 (Valderrama et al., 2019) y que más han impactado directamente en el campo de la enseñanza de la filosofía. En concreto, este texto analiza dos de las reformas recientes más relevantes en esta materia, a saber, las aplicadas a las licenciaturas y a las pruebas de Estado de la educación media.

El tono de este escrito es propio del trabajo de un observador, que aporta al seguimiento y construcción del campo de estudio de la enseñanza de la filosofía, en un esfuerzo que se suma al propósito emprendido por otros autores ${ }^{1}$. En los dos casos

1 Algunos de estos estudios nos permiten aproximarnos al campo de la historia de la enseñanza de la filosofia en Colombia. Al respecto, puede consultarse el estudio de José del Rey Fajardo y Germán Marquínez Argote (2010), acerca de la enseñanza de la filosofía en la Pontificia Universidad Javeriana 
mencionados describiremos el contenido de las políticas actuales, haremos un breve relato de la recepción que han tenido en las comunidades filosóficas y, posteriormente, presentaremos una reflexión sobre lo que, a nuestro juicio, está allí implicado para el desarrollo de la filosofía en el ejercicio de la enseñanza. Este último es el asunto que más nos interesa relevar, toda vez que las políticas públicas suelen ser síntomas de conceptos y tendencias subyacentes.

Adicionalmente, intentaremos relacionar los casos que aquí presentamos con la Declaración de Salvador de Bahía a Favor de la Filosofía ${ }^{2}$, con el propósito de mostrar que estos asuntos a los que Colombia se enfrenta son compartidos por la Red Iberoamericana de Filosofía, lo que revela que pueden ser tratados no solo como casos aislados, sino como preocupaciones de la región. Por consiguiente, este artículo también es un aporte a las discusiones que se presentan allende las fronteras nacionales en la materia señalada.

\section{La reforma a las licenciaturas}

En el año 2016, el Ministerio de Educación Nacional de Colombia (MEN) emitió la Resolución 2041, por medio de la cual “se establecen las características específicas de calidad de los programas de licenciatura para la obtención, renovación o modificación de registro calificado” (MEN, 2016, p. 1) , resolución que causó especial incomodidad

durante la Colonia. Asimismo, Saldarriaga (2008) y Montes y Montes (2019) estudian la filosofía como disciplina escolar en los siglos xIx y xx; i este último texto reporta en su bibliografía estudios particulares inéditos sobre periodos o procesos de la enseñanza de la filosofía en estos siglos. Un rastreo del campo de la enseñanza de la filosofía en cuanto teoría y práctica se encuentra en Vargas y Cárdenas (2004) y en Pulido, Espinel y Gómez (2018). Estudios de caso sobre los ajustes realizados por programas particulares en virtud de las resoluciones mencionadas se encuentran en Valderrama et al. (2019). El informe de la Unesco (2011), Filosofia: una escuela de la libertad, presenta, a su turno, el panorama latinoamericano y mundial acerca de la presencia de la filosofía en los niveles de educación básica, media y universitaria con datos que alcanzan la primera década del siglo xx.. El informe de la oel (1998) presenta un panorama de la presencia de la filosofía en las instituciones escolares iberoamericanas, con datos actualizados a la fecha de publicación del libro.

2 La Declaración de Salvador de Bahía a Favor de la Filosofía se dio a raíz del "I Encuentro de la Red Iberoamericana de Filosofia" realizado en la Universidade Federal de Bahía (UFBA) los días 19 y 20 de abril de 2017, en donde los representantes de redes nacionales e internacionales de filosofía de trece paises iberoamericanos se reunieron y realizaron una declaración para trabajar a favor de esta disciplina.

3 Es necesario aclarar que en la organización profesional colombiana se entienden por licenciatura únicamente los programas universitarios que preparan para la profesión docente, especialmente para la educación primaria y secundaria. 
entre las instituciones, estudiantes y profesores, al punto que, después de numerosas críticas provenientes de varios sectores, tal resolución tuvo que ser ajustada y fue derogada al año siguiente por la Resolución $18583 .{ }^{4}$

Quizás el aspecto que causó mayores inconformidades de tales resoluciones fue el referido a la alta dedicación a las prácticas pedagógicas en los programas de licenciatura, asunto que tiene como efecto indirecto la reducción del número de créditos dedicados a la disciplina específica; en nuestro caso, la filosofía. En la resolución 2041 de 2016 se pedía a las licenciaturas dedicar cincuenta créditos a las prácticas pedagógicas, mientras que la 18583 de 2017 redujo este número a cuarenta (Artículo 3.2). ${ }^{5}$ El asunto se ve agravado por el hecho de que la formación de un docente no implica solamente su ejercicio práctico, sino que incluye también otros componentes. Según tales resoluciones, los programas deben estar constituidos por cuatro componentes: el de fundamentos generales (lectura, escritura, argumentación, investigación, lengua extranjera, razonamiento cuantitativo, formación ciudadana y uso pedagógico de TICs), el de fundamentos específicos y disciplinares, el pedagógico y el didáctico (artículos 2.1 a 2.4); esto, sin contar que cada universidad suele dedicar otros créditos a la formación de carácter institucional. Adicionalmente, para fortalecer la formación pedagógica, las disposiciones ministeriales piden a las instituciones contar con investigación en áreas de enseñanza y con profesores con experiencia en aulas de educación básica y media.

Dado que las resoluciones fueron emitidas recientemente, aún no se cuenta con un diagnóstico completo de sus consecuencias en la estructura curricular, en la formación de docentes y en el ejercicio de la filosofía. Sin embargo, esto sí obligó a las universidades a realizar reformas inmediatas para cumplir con tales exigencias. Muchos de los programas de licenciatura realizaron ajustes mientras estuvo en vigencia la Resolución 2041 de 2016 que, recordemos, pedía cincuenta créditos de prácticas pedagógicas. Para estos programas, la amenaza de la reducción de la formación filosófica aún está vigente. Otros programas, sin embargo, pudieron realizarla cuando

4 A la fecha de redacción de este documento, la Resolución 18583 fue reemplazada por la 1330 de 2019.

5 En el sistema educativo colombiano, un crédito equivale a tres horas de trabajo semanal (cuarenta y ocho horas de trabajo al semestre). La enseñanza universitaria está organizada por semestres. Así, las carreras suelen tener una duración que oscila entre ocho y diez semestres. Cada semestre tiene una duración cercana a dieciséis semanas de ejercicio académico. 
entró en vigencia la Resolución 18583 de 2017, que exigía cuarenta créditos para tal aspecto. Esta última matizó el panorama desalentador frente a la escasa formación filosófica de los licenciados. Las distintas alternativas que tomaron las universidades para hacer frente a esto fueron desde aumentar la cantidad de créditos de la carrera - y con ello el número de semestres - hasta cerrar los programas o desplazarlos de facultad: en lugar de ofrecerlos en las facultades de filosofía, algunas universidades los trasladaron a las facultades de educación, pues en ocasiones estas serían las únicas que estarían en condiciones de dar soporte investigativo y pedagógico a los programas, según las exigencias del MEN.

Es necesario volver sobre los conceptos y principios que fundamentan el crecimiento de la formación pedagógica en los docentes. El establecimiento de los créditos como indicadores es resultado de tendencias generales, pero ¿en qué se sustenta esta orientación del MEN? Los análisis previos a la formulación de las reformas señalan que el elemento clave para consolidar una "Colombia más educada”, según el lema del Plan Nacional de Desarrollo (PND) 2014-2018, se encuentra en los docentes: en sus condiciones de contratación, en su formación inicial y a lo largo de la vida, así como en el seguimiento y evaluación que se hace de su desempeño. Como muestran Arias et al. (2018), este hecho es patente en el PND: "se resalta que los indicadores de la educación básica dependen de la calidad docente, pues los resultados en las pruebas Saber se relacionan con docentes con mejor formación previa o menor proporción de estos en condición de proporcionalidad" (pp. 89-90). La mejor formación inicial se comprendió, a su turno, como reconocimiento de que el saber fundante del docente es la pedagogía, con lo cual se le reconoce, a su vez, como profesional de la educación (Arias et al., 2018, p. 99). En consecuencia, como lo muestran estos autores, las políticas de formación de docentes apuntan al fortalecimiento del saber enseñar, visto como un ejercicio práctico fundado en componentes pedagógicos y didácticos (Arias et al., 2018, p. 98).

Dada esta premisa inicial, el aumento de créditos para la práctica pedagógica, tal como lo pedían las resoluciones reseñadas, no es sino una consecuencia necesaria, aunque quepa, no obstante, la crítica acerca de si declarar la pedagogía como saber fundante conduzca necesaria y exclusivamente al fortalecimiento de la didáctica o a la implementación de un modo instrumental de comprender la pedagogía y la didáctica. Así, aunque se haya disminuido la exigencia de créditos de cincuenta a cuarenta, 
la premisa que sustenta este mandato se mantiene intacta, por lo cual se esperan nuevas consecuencias.

Ahora bien, aunque en términos de derechos laborales, de constitución de un gremio, de reconocimiento del saber pedagógico y, fundamentalmente, para el mejoramiento de la enseñanza en la escuela, las orientaciones y normativas reseñadas constituyen un avance significativo, desde el punto de vista disciplinar se experimentan consecuencias adversas importantes. Por ello, en lo que sigue, nos aproximaremos a las reacciones de las comunidades filosóficas ante estas.

La Red Nacional de Programas de Filosofía se reunió el 31 de mayo de 2017 en la ciudad de Medellín con el propósito de realizar un primer diagnóstico de los efectos de tales resoluciones en los programas. Al respecto, una de las conclusiones señala lo siguiente:

Aunque se reconoce que el aumento de número de créditos en prácticas pedagógicas fortalece la formación en pedagogía de los licenciados, este hecho, sumado a las exigencias de incluir en la formación competencias básicas y alto nivel de idiomas, hace que se afecte la formación disciplinar, en nuestro caso, de la filosofía. Así, tales exigencias ponen en riesgo el equilibrio necesario que se requiere entre la formación disciplinar y la pedagógica en la formación de un licenciado, un asunto especialmente sensible en las licenciaturas que vinculan más de un área disciplinar. No es posible formar maestros sin una rigurosa formación e investigación, tanto en la disciplina como en pedagogía. (Conclusiones de la reunión del 31 de mayo de 2017, sostenida en la Universidad de Antioquia, Medellín) ${ }^{6}$

Valderrama et al. (2019, p. 83) han llegado a conclusiones similares a partir de tres estudios de caso sobre el impacto de tales resoluciones en la organización curricular de los programas de filosofía, en lo que denominan el “arrinconamiento" del componente disciplinar que causó la reforma. La consecuencia podría ser el deterioro de la

6 Nótese que a la fecha de celebración de este encuentro no había sido emitida aún la Resolución 18583 de 2017, publicada el 15 de septiembre. De manera que esta reunión se realizó sobre la base de la 2041 de 2016 que exigia cincuenta créditos de práctica pedagógica. Hay que advertir que aunque aquella derogó esta última, la necesidad de ajustar los planes de estudio a la 2041, pues se exigía en la misma resolución cambios inmediatos, hizo que se reformaran los programas según tales exigencias y no según las de la 18583. 
formación filosófica entre la mayoría de los profesores de esta disciplina — según las alternativas que haya tomado cada institución para hacer frente a las resoluciones-, pues el reducido número de créditos dedicado a esta en la formación implicaría que de ella se tuviera apenas una mirada "panorámica y generalizante" (Valderrama et al., 2019, p. 88), en aras del fortalecimiento de la formación pedagógica de los futuros maestros.

En este sentido, una de las consecuencias diagnosticadas en las comunidades filosóficas se refiere a la presencia de las disciplinas en la formación de licenciados. En efecto, una vez se establece que el saber fundante del docente es la pedagogía, la disciplina que enseña pasa a un segundo plano. En dicho escenario, esto es, en la formulación de las políticas públicas, la formación disciplinar apenas ocupa un lugar relevante; se presenta como un saber incidental, toda vez que las distinciones entre ser docente de una u otra área tienden a disiparse. De hecho, la Resolución 18583 se refiere a las disciplinas como los campos en los que los licenciados se desempeñan (Artículo 2.2.), esto es, las disciplinas pasan a ser especificidades o desarrollos dentro del saber general: la pedagogía.

No se trata solo de la disputa gremial por un campo de ejercicio y saber. En lo que respecta a la enseñanza de la filosofía, queremos destacar dos aspectos. El primero de ellos consiste en que esta sitúa los principios de su enseñanza por fuera de la construcción de la disciplina, lo que genera un abismo entre la producción filosófica y su enseñanza. La filosofía ha mantenido una relación estrecha con la formación, al punto que en algunos momentos de la historia se le comprendía como proceso formativo ${ }^{7}$. Además, la filosofía ha tendido a encontrar en ella misma las bases y fundamentos de su "enseñabilidad". El curso mismo del filosofar, en sus distintas corrientes y métodos, ha sido la base de la enseñanza y el aprendizaje, considerando que enseñar y aprender filosofía no son un asunto ajeno al filosofar mismo. Siguiendo este orden de ideas, el docente de filosofía ha sido reconocido como profesional de esta área específica. Así, una separación entre la construcción filosófica y su enseñanza tendría como consecuencia que el contenido a ser enseñado se alejaría de la generación de conocimiento, toda vez que se investiga siguiendo métodos y criterios internos, pero se enseñaría

7 La bibliografía a este respecto es abundante. Resultaria prolijo hacer un recuento de esta tendencia. Baste decir, por el momento, que esta premisa constituyó la base teórica de un número especial de la revista Pedagogía y Saberes en 2017. Así mismo, la perspectiva de filosofía como forma de vida refleja esta idea (Hadot, 1998; 2006; Calvo Ortega, 2010). 
bajo otros parámetros. Dicho sea de paso, la desarticulación entre investigación y enseñanza conllevaría la fractura entre comunidades filosóficas (universidad y escuela). Esto no implica, desde luego, que la filosofía se baste a sí misma cuando se trata de su enseñanza. En una posición extrema y errada, hay que decirlo también, se ha llegado a considerar que saber filosofía implica saber enseñar o que esta tiene recursos suficientes para garantizar procesos de enseñanza de calidad, lo que desconoce los aportes de la pedagogía, la didáctica y otras disciplinas al campo de la educación. En nuestro caso, abogamos porque la enseñanza requiere saberes fundantes: la disciplina y la pedagogía.

Por otro lado, esta Resolución dificulta el que se lleven a cabo los objetivos por los cuales se considera pertinente la enseñanza de la filosofía en la escuela. Esto es, si la enseñanza de la filosofía se justifica porque contribuye a la formación ciudadana, a la construcción de la paz o al desarrollo de la autonomía y el pensamiento crítico (Nussbaum, 2010; Vargas, Meléndez y Herrera, 2017), entre otros aspectos, no se explica de qué manera, con una presencia incidental de esta en los procesos educativos se podrían alcanzar dichos objetivos. Aun cuando en el currículo sigan teniendo cabida las materias de filosofía, es necesario hacer de ellas espacios del filosofar y no solamente escenarios donde se apliquen estrategias para alcanzar resultados exitosos en las pruebas estandarizadas o donde solo se brinde información sobre la historia de la filosofía. A pesar de que existan materias filosóficas, es necesario que estas sean espacios de ejercicio profesional de la filosofía. En esta dirección apuntan la Unesco y la Red Iberoamericana de Filosofía cuando en sus comunicaciones oficiales señalan: "Su enseñanza [de la filosofía] debe mantenerse o ampliarse donde ya existe, implantarse donde aún no existe y ser nombrada explícitamente con la palabra 'filosofía" (Unesco, 2009, p. 123).

Por eso, una sociedad democrática debe contar, entre otros requisitos imprescindibles, con un sistema público de educación que cubra todas las etapas formativas (infantil, primaria, secundaria y universitaria), que proporcione una formación de calidad, que sea accesible a todas las personas sin discriminación alguna por razón de sexo, nacionalidad, clase social, ideología, lengua, religión, etc., y que conceda la debida importancia a los estudios de filosofía, denominada explícitamente como tal y enseñada por filósofos profesionales, desde la infancia hasta la educación superior. (Declaración de Salvador de Bahía, numeral 5) 
En efecto, ¿qué ha ocurrido con la formación filosófica en los programas universitarios de filosofía?, ¿es accidental o se encuentra arrinconada? Consideremos el estado actual de los programas de formación universitaria en filosofía, en concreto, el problema que hemos rastreado y que abordaremos a partir de una aproximación a la distribución del número de créditos.

Los datos que reportan las universidades en el Sistema Nacional Integrado de Educación Superior (sNiEs), tanto como los planes de estudio de cada uno de los programas universitarios de formación en filosofía permiten reconstruir la situación descrita hasta el momento. En efecto, una aproximación a la distribución del número de créditos de los programas revela el peso específico que tienen la filosofía y la pedagogía, entre otros componentes, en la formación de los profesionales.

Según el SNIES, a junio de 2019, en Colombia existen 57 programas de formación universitaria en filosofía. Dentro de ellos, 27 son licenciaturas ${ }^{8}$ y 30 otorgan título de profesional en filosofía o similares. ${ }^{9}$ Las licenciaturas ofrecen tres titulaciones: Licenciado en Filosofía (20 programas), Licenciado en Filosofía y Letras (5) y Licenciado en Filosofía y Educación Religiosa (2). Por su parte, los restantes 30 programas distribuyen su titulación así: Profesional en Filosofía o Filósofo (24 programas), Profesional en Filosofía y Humanidades (2), Profesional en Filosofía y Letras (3) y Profesional en Filosofía y Teología (1).

Actualmente, el conjunto de los 57 programas de filosofía consta de entre 122 y 174 créditos de formación. Por una parte, las licenciaturas presentan un promedio de créditos de 157 (el programa que menos créditos ofrece es 122 y el que más 174; la oferta de créditos recurrente - moda - es 160), mientras que en el conjunto de los restantes 30 programas el promedio es de 141,3 (el programa que menos créditos ofrece es 122 y el que más 170; la oferta de créditos recurrente — moda- es 136).

8 La misma Resolución 2041 de 2016 que hemos citado, en su Artículo 2, define las denominaciones aceptadas por la nación en relación con las licenciaturas específicas de filosofía. Esta admite solamente: Licenciatura en Filosofía, Licenciatura en Filosofía y Letras y Licenciatura en Ética y Valores. Cabe aclarar que los programas de licenciatura también son profesionales, en cuanto su titulación es otorgada por una universidad y no tienen un carácter técnico ni tecnológico.

9 Las titulaciones que se dan en el pais son: Profesional en Filosofía, Filósofo, Profesional en Filosofía y Humanidades, Profesional en Filosofía y Letras y Profesional en Filosofía y Teología. 
Pero, si bien las licenciaturas cuentan con un mayor número de créditos totales, los programas de titulación profesional dedican una mayor cantidad de créditos al componente filosófico que las licenciaturas. Es así que en las licenciaturas el total de créditos destinados al componente filosófico es aproximadamente 75 , equivalente al $47,84 \%$ de formación ${ }^{10}$; en tanto que en los programas de titulación profesional el componente filosófico es de cerca de 109 créditos, equivalente al 77,26\% (la oferta recurrente - moda - es 116 créditos de filosofía). En las licenciaturas, se dedican adicionalmente en promedio 50 créditos al componente pedagógico (32,02\%) y cerca de $20,14 \%$ a otros componentes de la formación. En el caso de los programas de profesional en filosofía, estos otros componentes constituyen el restante porcentaje de créditos, esto es, $22,67 \%$.

Lo anterior indica que en promedio las licenciaturas en filosofía siguen dedicando mayor tiempo a la formación disciplinar, frente a otros componentes. En el presente texto no nos es dado valorar si el promedio de 79 créditos de formación inicial en filosofía es suficiente para formar un docente de alto nivel, ${ }^{11}$ pues, por un lado, esto requeriría acercar la mirada al contenido específico de los planes de estudio y a los enlaces interdisciplinares que se dan en su interior, ampliar el objeto a elementos que vayan más allá del número de créditos de los programas y examinar resultados que solo se pueden estudiar utilizando perspectivas temporales. Como indicamos al inicio del artículo, nuestro punto de vista es el de un observador que reporta y mediante la investigación intenta explicitar el sentido de los movimientos que se dan en el campo de la enseñanza de la filosofía. Cabe señalar que, no obstante, existe una diferencia de cerca de 30 créditos entre la formación filosófica de las licenciaturas y la de los restantes programas disciplinares; la otra cara de esta diferencia es que las licenciaturas destinan aproximadamente 50 créditos a la formación pedagógica, mientras que no lo hacen así los programas profesionales. ${ }^{12}$

10 La cifra $47,84 \%$ corresponde al porcentaje de los créditos filosóficos en relación con el promedio de créditos que tiene el conjunto de las 27 licenciaturas (157 créditos). Si se toma en cuenta solamente el conjunto de las Licenciaturas en Filosofía (20 programas), y no las que forman en filosofía y otras áreas, el porcentaje sería de $51 \%$.

11 La situación podría agravarse en el caso de los programas en donde se forma en más de una disciplina.

12 El debate acerca de si la enseñanza de la filosofía debe estar en manos solamente de licenciados supera los límites de este artículo. Nuestro interés aquí es mostrar una de las tesis fundamentales de la enseñanza de esta disciplina: aquella según la cual para enseñar filosofía hay que saber filosofía. 
Las universidades han reaccionado de diversas maneras a las resoluciones mencionadas. Al parecer, la mejor alternativa para que no se reduzca la filosofía en la formación de docentes ha sido hasta ahora la de aumentar el número de semestres de duración de las carreras, como ya se mencionó, con lo cual se puede mantener una cantidad adecuada de créditos disciplinares. En efecto, actualmente el promedio de duración de las licenciaturas es de 9,3 semestres académicos (la duración más recurrente - moda - es 10 semestres), mientras que el promedio de los restantes 30 programas profesionales es de 8 (esta misma cifra es la oferta recurrente). Sin embargo, esto le resta competitividad a los programas, pues la tendencia general de la formación universitaria es desarrollarlos en 8 semestres. Los programas de formación de docentes se encuentran en esta encrucijada. Aun así, aunque la reducción de créditos destinados a la filosofía en la formación de docentes sea resuelta de esta manera, queda pendiente la discusión acerca de la conformación y saber fundante de un docente de filosofía y, en consecuencia, su estatus profesional.

\section{Las pruebas Saber 11}

En Colombia, al finalizar la educación secundaria (en el grado 11), los estudiantes realizan una prueba estandarizada de Estado, denominada prueba Saber 11, diseñada por el Instituto Colombiano para el Fomento de la Educación Superior (Icfes). Al ser estandarizada, sirve para situar en un marco nacional los alcances obtenidos por cada estudiante, pero también, a partir de los resultados individuales se evalúa el desempeño de la institución de la cual egresa. Esta prueba se aplica desde 1980, e incluía el área de filosofía, sin embargo, en lo que aquí nos interesa rastrear (la enseñanza de la filosofía) debemos detenernos en la reforma que se hizo a esta prueba en el año 2000, toda vez que a partir de entonces la prueba dejó de enfocarse en evaluar contenidos y pasó a evaluar competencias (Icfes, 2013, p. 15). El segundo hito que nos interesa se dio en el año 2014, cuando se dejó de evaluar el área de filosofía de modo diferenciado y se fusionó con el de lenguaje, para constituir la prueba de lectura crítica. La documentación que presenta esta reforma determinó:

Fusionar las pruebas de Lenguaje y de Filosofía en una prueba de Lectura Crítica. Por un lado, desde la reestructuración del examen realizada en el año 2000 la prueba de Filosofía está orientada hacia la evaluación de competencias de lectura crítica. No exige conocimientos propios de la historia de la filosofía, y los conceptos filosóficos involucrados se explican brevemente en la formulación 
de las diferentes preguntas. Por otro lado, la prueba de Lenguaje evalúa competencias que, al final de la educación media, deben haber alcanzado el nivel propio de la lectura crítica. La diferencia entre las pruebas de Lenguaje y de Filosofía concierne entonces únicamente al tipo de textos que se utilizan: textos filosóficos en la prueba de filosofía. (Icfes, 2013, p. 27)

Después de esto, el documento detalla lo que entiende por lectura crítica:

Para aproximarse críticamente a un texto un estudiante debe, en primer lugar, comprender las unidades locales de sentido. En segundo lugar, debe integrar esa información para darle un sentido global al texto. Y, en tercer lugar, una vez superadas las dos etapas anteriores, debe tomar una postura crítica frente al texto, reflexionando sobre su contenido. (Icfes, 2013, p. 42)

Al igual que las resoluciones que reformaron las licenciaturas, los cambios en las pruebas Saber 11 motivaron reacciones diversas de distintos actores. Quizá los más vehementes frente a este particular fueron la Comisión de Enseñanza de la Filosofía de la Sociedad Colombiana de Filosofía y algunas universidades. ${ }^{13}$ A partir de foros, publicaciones académicas, escritos y comunicaciones en distintas plataformas, entre otras manifestaciones, se demandó que medidas como esta impactan negativamente el desarrollo de la filosofía, al punto de señalar que tales reformas se suman al "asesinato de la filosofía" que se venía experimentando en el país. ${ }^{14}$

Dentro de la Ley General de Educación (Ley 115 de 1994, Artículo 3), que establece las asignaturas que componen el currículo escolar, la filosofía sigue estando presente como área fundamental de la educación media académica. ¿Por qué se habla, entonces, de asesinato de la filosofía si aún existe esta área en la escuela?

13 Entre estas se encontraron la Universidad Pedagógica Nacional, la Universidad del Rosario, la Universidad Santo Tomás y la Universidad Nacional de Colombia, entre otras.

14 Esta expresión fue utilizada en el documento titulado "Manifiesto contra el asesinato de la fiIosofía en Colombia", elaborado por la Licenciatura en Filosofía de la Universidad Pedagógica Nacional en septiembre de 2014, como respuesta a tales cambios en las pruebas Saber 11. El manifiesto puede ser consultado en: https://profesorvargasguillen.com/2014/09/22/ manifiesto-contra-el-asesinato-de-la-filosofia-en-colombia/ Dos comentarios a este, elaborados por profesores de distintos lugares, se encuentran en: https://profesorvargasguillen. com/2015/01/28/carta-abierta-a-los-maestros-de-filosofia/ y https://www.academia.edu/11816682/ Respuesta_al_manifiesto_contra_el_asesinato_de_la_filosof\%C3\%ADa_en_Colombia 
Tal expresión fue utilizada en relación con la importancia de la prueba Saber 11 para la educación media. ${ }^{15}$ En efecto, aunque se pueda argumentar que los propósitos de fomentar la lectura crítica pueden ser razonables para el área de filosofía, al tratarse de una prueba estandarizada, a partir del PND de 2006, el Estado se concibe como árbitro de la dinámica social (Martínez, Noguera y Castro, 2003, p. 207), asunto que se concretó en que en materia educativa el Estado asume la calidad en función de los resultados de las pruebas estandarizadas, perspectiva que se mantiene incluso en el PND 2014-2018 (Arias et al., 2018, p. 89). Siguiendo esta directriz, las pruebas Saber 11 son el referente fundamental de evaluación de estudiantes, de instituciones y del proceso formativo en su conjunto. De allí que, en consecuencia, algunos aspectos de la financiación de unos y otros están enlazados a tales resultados. ${ }^{16}$

Además de la presión que ejercen las pruebas estatales sobre las instituciones educativas y los estudiantes, también tienen efectos en la presencia y sentido de la enseñanza de la filosofía: concordamos con el "Manifiesto contra el asesinato de la filosofía en Colombia" (Licenciatura en Filosofía UPN, 2014), en que se utiliza la expresión "asesinato" en cuanto la eliminación de la filosofía en la prueba la diluye en función de propósitos externos y la reduce a una función instrumental. ${ }^{17}$ En efecto, si las instituciones educativas pretenden ser reconocidas por su calidad y, en consecuencia, recibir más recursos para su financiación, lo cual se logra al obtener resultados altos en estas pruebas, la filosofía ya no es necesaria, salvo por su aporte a la lectura crítica. El desarrollo de otros contenidos, métodos o competencias dentro de esta área se empieza a ver como accesorio a la formación, máxime cuando a la filosofía se le dedican una o dos horas lectivas semanales. Como señala el manifiesto citado: "Eliminada la filosofía de la medición final en la formación básica, consecuentemente se hace inocua su enseñanza en todo el proceso que precede al examen" (Licenciatura en Filosofía UPN, 2014, p. 5). En consonancia con ello, se desdibuja la idea presente en el numeral 4 de la Declaración de Salvador de Bahía (oIss, 2004), donde se afirma que:

15 Una consecuencia previsible del aumento del valor de la prueba Saber 11 en las instituciones educativas es el olvido de otras fuentes que sustentan los contenidos y orientaciones de la formación filosófica, como las Orientaciones pedagógicas para la filosofía en la educación media de Colombia (MEN, 2010).

16 A este respecto vale la pena mencionar el programa estatal Ser Pilo Paga, a través del cual se otorga una beca-crédito condonable para ingresar a la educación superior a los estudiantes de escasos recursos que obtengan los resultados más altos en estas pruebas.

17 Para profundizar en este tema se recomienda el artículo de Prada y Acevedo (2017, pp. 15 y ss.). Asimismo, sobre la función de la lectura como aporte ético en la formación, ver Vargas, Herrera y Meléndez (2017, p. 69). 
[...] los estudios de filosofía deben tener un lugar específico y diferenciado en el sistema educativo, porque solo de ese modo se garantiza su transmisión generacional y su renovación permanente, y porque toda persona tiene "derecho a la filosofía", es decir, derecho a recibir una formación básica que le permita acceder al rico patrimonio del pensamiento filosófico y, en caso de que lo desee, adquirir también los conocimientos especializados necesarios para el ejercicio de la profesión y vocación filosóficas. (Licenciatura en Filosofía UPN, 2014, p. 5)

Reducida a instrumento de lectura, la filosofía apenas podría cumplir con los objetivos altos que están en la base o que justifican su presencia en la educación media; los cuales, como hemos mencionado, se refieren fundamentalmente a la formación democrática, ciudadana y para la paz.

Las distintas reacciones frente a la composición de la prueba derivaron en el establecimiento de un diálogo y trabajo mancomunado entre la comunidad académica de filosofía y el Icfes, para garantizar que, en efecto, las preguntas de lectura crítica tuvieran un alto contenido de textos filosóficos.

Por otra parte, las universidades, quizá como crítica al enfoque que exigen las pruebas, solo tímidamente se han enfocado en la formación filosófica orientada a la lectura crítica. Por el contrario, para minimizar los efectos del posible desinterés de las instituciones en la enseñanza de esta disciplina, se hace necesario un acercamiento entre las universidades y la educación básica y media, de modo que tal enlace brinde a las escuelas y maestros los apoyos necesarios para continuar con la formación filosófica, dado que ya no existen tales apoyos por vía de las pruebas estatales.

\section{Conclusiones}

No conviene perder de vista el proceso en el cual se justifica, en la actualidad, la enseñanza de la filosofía en Colombia: la consolidación de una democracia y de un país en paz. Como señalan Vargas, Meléndez y Herrera (2017), quizás el reto más importante no se encuentra en su desarrollo intrínseco sino en su relación con el momento histórico:

[...] quizá lo más importante, en relación con lo que se ha tratado, es que el esfuerzo central de la filosofía está en analizar los problemas fundamentales 
de cada presente histórico. Al estar en Colombia, en donde sale a la palestra por doquier la barbarie en que los ciudadanos han vivido en todos estos años, una cuestión urgente para la formación de estos es ¿cómo hacer frente a los desacuerdos, que como democracia no simplemente se deben aceptar sino celebrar, sin acudir a la violencia o al acallamiento de la deliberación reflexiva? (Vargas, Meléndez y Herrera, 2017, p. 70)

Pero, aun cuando la formación ciudadana y para la paz sea el objetivo más importante en lo que se refiere a la educación básica y media, la filosofía no ha encontrado su lugar dentro de los propósitos y acciones que expresan las normas nacionales recientes sobre los procesos educativos. El énfasis en la medición de la calidad no parece ser compatible con los propósitos que la filosofía considera pertinentes para nuestra sociedad. Este desencuentro revela que urge un diálogo entre la comunidad filosófica, la pedagogía y el país, en este caso, en torno a la promoción de políticas públicas que vinculen tanto los objetivos de la formación filosófica como las apuestas pedagógicas que requiere la enseñanza.

En nuestro caso, como intentamos mostrar en este escrito, el asunto a discutir no ha sido el de la presencia o no de materias filosóficas en la estructura curricular escolar, tal como fue la tendencia marcada por Nussbaum (2010) y que ha tenido eco en varios países latinoamericanos a raíz de reformas que han propuesto retirar las materias filosóficas del currículo. Se prevé que ese asunto debe ser discutido prontamente en el país, así como otros tópicos que se encuentran de la formación filosófica: libros de texto en la enseñanza de la filosofía, orientaciones curriculares, formación ética y cátedra de paz, entre otros. En su lugar, el ser observadores nos ha llevado a centrar la mirada en el conocimiento del profesor que ocupa esos espacios y el de las orientaciones y sentido que toma el área de la filosofía en la educación media, es decir, en cuestiones que configuran el espacio de esta materia en la escuela. Las dos cuestiones, a su turno, están relacionadas: en efecto, si el área de filosofía no es orientada por un egresado universitario con amplio conocimiento de la disciplina es previsible que se reduzca el sentido de su enseñanza; en el mejor de los casos, hacia la lectura crítica, o, como ha ocurrido en ocasiones, a repetir contenidos extraídos de manuales o entornos virtuales que no apuntan al filosofar mismo. Urge, pues, un diálogo entre filosofía y pedagogía para enriquecer la enseñanza de esta disciplina. 


\section{Referencias}

Arias, G., Diego, H., Díaz, F., Olga, C., Garzón Barragán, I., León Palencia, A., Rodríguez Ávila, S. P. y Valbuena Ussa, E. (2018). Entre las exigencias de calidady las condiciones de desigualdad: formación inicial de profesores en Colombia. Bogotá: Colciencias y Universidad Pedagógica Nacional.

Calvo Ortega, F. (2010). Ejercicios espirituales y pedagogía: la práctica de la filosofía en el mundo grecolatino. En A. Moreu y E. Prats (coords.), La educación revisitada: ensayos de hermenéutica pedagógica (pp. 39-58). Barcelona: Universidad de Barcelona.

Congreso de la República de Colombia. (1994). Ley 114 de 1994: Ley General de Educación. Recuperado de https://www.mineducacion.gov.co/1621/ articles-85906_archivo_pdf.pdf

Del Rey Fajardo, J. y Marquínez Argote, G. (2010). La enseñanza de la filosofía en la Universidad Javeriana colonial (1623-1767). Bogotá: Pontificia Universidad Javeriana.

Instituto Colombiano para el Fomento de la Educación Superior (Icfes). (2013). Sistema Nacional de Evaluación Estandarizada de la Educación: Alineación del Examen Saber 11. Bogotá: Ministerio de Educación Nacional.

Hadot, P. (1998). ¿Qué es la filosofía antigua? E. Cazenave (trad.). Madrid: Fondo de Cultura Económica.

Hadot, P. (2009). Ejercicios espirituales y filosofía antigua. Madrid: Siruela.

Licenciatura en Filosofía, Universidad Pedagógica Nacional de Colombia (UPN). (2014). Manifiesto contra el asesinato de la filosofía en Colombia. Recuperado de https://profesorvargasguillen.com/2014/09/22/manifiesto-contra-el-asesinatode-la-filosofia-en-colombia/

Martínez Boom, A., Noguera, C. y Castro, J. (2003). Currículo y modernización. Cuatro décadas de educación en Colombia. Bogotá: Magisterio.

Ministerio de Educación Nacional (MEN). (2010). Orientaciones pedagógicas para la filosofía en la educación media. Recuperado de https://www.mineducacion.gov. co/1621/articles340033_archivo_pdf_Orientaciones_Pedagogicas_Filosofia_en_ Educacion_Media.pdf 
Ministerio de Educación Nacional (MEN). (2016). Resolución 2041 de 3 de febrero de 2016. Recuperado de http://www.alcaldiabogota.gov.co/sisjur/normas/Norma1. jsp?i=71388\#2041

Ministerio de Educación Nacional (MEN). (2016). Resolución 18583 de 15 de septiembre de 2017. Recuperado de http://www.alcaldiabogota.gov.co/sisjur/normas/ Norma1.jsp?i=71384

Montes Montes, V. D. y Montes Montes, J. A. (2019). La filosofía como disciplina escolar en Colombia 1946-1994. Bogotá: Aula de Humanidades.

Nussbaum, M. (2010). Sin fines de lucro. Por qué la democracia necesita de las humanidades. Buenos Aires: Katz.

Organización de Estados Iberoamericanos (OEI). (1998). Análisis de los currículos de filosofía en el nivel medio en Iberoamérica. Madrid.

Organización Iberoamericana de Seguridad Social (oIss). (2004). Declaración de Salvador de Bahía. Salvador de Bahía.

Prada, M. y Acevedo, D. (2017). Pensar la vida: crisis de las humanidades y praxis filosófica. Revista Colombiana de Educación, 72, 15-37.

Pulido, O., Espinel, O. y Gómez, M. (Coord.). (2018). Filosofía y enseñanza: miradas en Iberoamérica. Tunja: Universidad Pedagógica y Tecnológica de Colombia.

Saldarriaga, O. (2008). De universidades a colegios: la filosofía escolar y la conformación del bachillerato moderno en Colombia, 1792-1892. En S. Castro Gómez y E. Restrepo (comps.), Genealogías de la colombianidad. Formaciones discursivas y tecnológicas de gobiernos en los siglos XIX y XX (pp. 308-333). Bogotá: Pontificia Universidad Javeriana.

Valderrama L., Gómez, M., Murillo, L. A., Farieta, R. A. y Vela, L. M. (2019). Licenciaturas en filosofía: disputas y articulaciones entre lo disciplinar y lo pedagógico. En M. Beltrán, C. Cáceda y L. Murillo (comps.), Formación de docentes en universidades latinoamericanas (pp. 71-111) Bogotá: Uniagustiniana.

Vargas, G. y Cárdenas, L. G. (2004). Filosofía, pedagogía y enseñanza de la filosofía. Bogotá: Universidad Pedagógica Nacional. 
Vargas, G., Meléndez, R. y Herrera, W. (2017). Experiencia y problemas. Educación ciudadana y enseñanza de la filosofía. Pedagogía y Saberes, 47, 65-77.

Red Nacional de Programas de Filosofía. (2017). Conclusiones de la reunión del 31 de mayo de 2017. Medellín: Universidad de Antioquia. Sin publicar.

Unesco. (2009). La enseñanza de la filosofía en América Latina y el Caribe: memorias de la Reunión Regional de Alto Nivel. Santo Domingo: Unesco.

Unesco. (2011). La filosofía, una escuela de la libertad. Enseñanza de la filosofía y aprendizaje del filosofar: la situación actual y las perspectivas para el futuro. París: Unesco. 